\title{
Experimental Study on the Influence of Wetting-Drying Cycle on the Strength Characteristics of Expansive Rock
}

\author{
JIA Xiaobing ${ }^{1,2, a}$, ZHAO Erpng ${ }^{1, b}$ \\ ${ }^{1}$ Key Laboratory of Geological Hazards on Three Gorges Reservoir Area(China Three Gorges \\ University),Ministry of Education, Yichang \\ ${ }^{2}$ Three Gorges Vocational College of Electric Power, Yichang \\ axc jxb@163.com, ${ }^{\mathrm{b}} \underline{58517507 @ q q . c o m}$
}

\begin{abstract}
Keywords: expansive rock, wetting-drying cycle test, strength characteristics
Abstract. After absorbing water, expansive rock will expand and soften. After losing water, expansive rock will shrink and crack. The season alternates, expansive rock repeatedly expands and shrinks, which has potential hazard to the project. So, in order to study the influence of wetting-drying cycle on the strength characteristics of expansive rock, the in-room cyclic wetting-drying test of expansive rock samples were carried out. The results showed that the cohesion c decreases greatly after the first wetting-drying process, the cohesion $\mathrm{c}$ attenuated by $37.1 \%$. In the following wetting-drying processes, the decreasing rate reduced. The friction Angle decreased greatly in the first cycling, fluctuated afterwards ,but the total value didn't show a great variation.
\end{abstract}

\section{Introduction}

Expansive rock is a special gneiss, it is extreme sensitivity to temperature, humidity and stress field. After absorbing water, expansive rock will expand and soften. After losing water, expansive rock will shrink and crack. The strength changes a lot. Zeng Jijie[1] studied the engineering characteristics, the stability of the slope, and engineering measures of expansive mudstone in the Nanning basin. Zang Deji[2] studied the shear behaviors of undisturbed sample. He Xiaomin etc.[3]studied the physical and mechanical character of swelling rock which supplied the scientific reference for the design and construction of swelling rock slope in Middle-Route of the South-to-North Water Transfer Project. Hou Xuemei [4] studied the General strength and swell-shrink characteristics of expansive rock at the base of indoor experiments. The results showed that the strength parameters of expansive rock were related with moisture content. The fresh rock surface after excavation would soon have a fracture, and the extent of cracking by temperature and humidity influence. XU Han etc. [5] studied the strength characteristics of expansive rock. The results showed that the less initial water content was, the larger competence of absorbing water became and the larger strength reduction degree was, also the smaller saturation strength parameter was. When the water content of sample was low, its strength was big but the failure strain was small.

But with the season alternating, the expansive rock repeatedly expand and shrink, which has great influence on the strength of expansive rock. As a result, the article studied the influence of wetting-drying cycle on the strength characteristics of expansive rock.

\section{Test design}

First, the in-room cyclic wetting-drying test of expansive rock samples were carried out. Then, direct shear tests of the expansive rock were carried out, which can obtained the strength, cohesion and the internal friction angle of the expansive rock after the cyclic wetting-drying tests. The static pressure formed expansive rock samples were used in this experiment.

The cyclic wetting-drying test: a total of 4 cycles. The initial water content of the sample was $14.8 \%$ and the dry density was $1.89 \mathrm{~g} / \mathrm{cm}^{3}$. A total of 5 samples were prepared in this experiment. After the samples were prepared, the samples were saturated with the vacuum pumping method, and the vacuum pumping time was 1 hour, and the soaking time of the samples in the vacuum was 12 
hours. Then, took out 4 samples, and conducted the direct shear test. Then, took out the rest samples, put the samples in the incubator (temperature $80^{\circ} \mathrm{C}$ ), drying time was 10 hours. Then, saturated the samples with the vacuum pumping method. After saturating the samples, took out 4 samples, and conducted the direct shear test. After that, four wetting-drying test cycles were carried out. Then, the tests were completed.

The rapid direct shear test of expansive rock was carried out on the conventional strain controlled direct shear apparatus. The vertical pressures are $50 \mathrm{kPa}, 100 \mathrm{kPa}, 150 \mathrm{kPa}, 200 \mathrm{kPa}$. The shear rate is $0.8 \mathrm{~mm} / \mathrm{min}$.

\section{Analysis of test results}

The relationship between vertical stress and shear stress of expansive rock under different wetting-drying cycle times was shown in Fig. 1. With the index of shear strength as the vertical coordinate and the number of dry and wetting-drying cycle times as the horizontal coordinates, the variation of the index of the strength of the expansive rock shear strength with the wetting-drying cycle times was shown in Fig. 2.

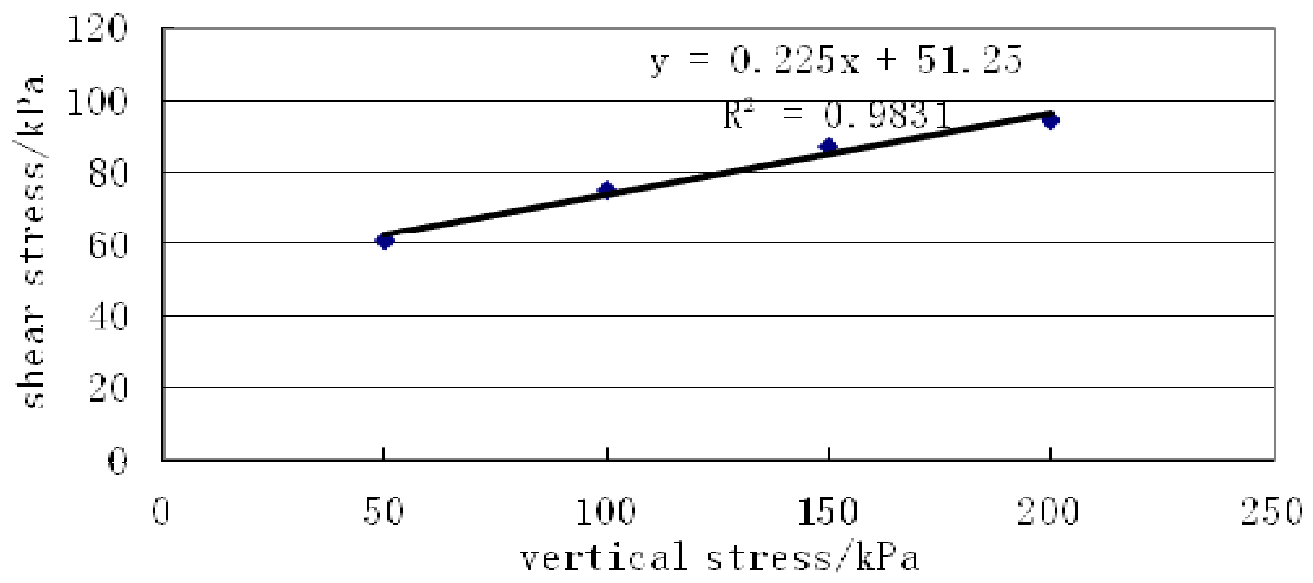

(a) 0 time wetting-drying cycle

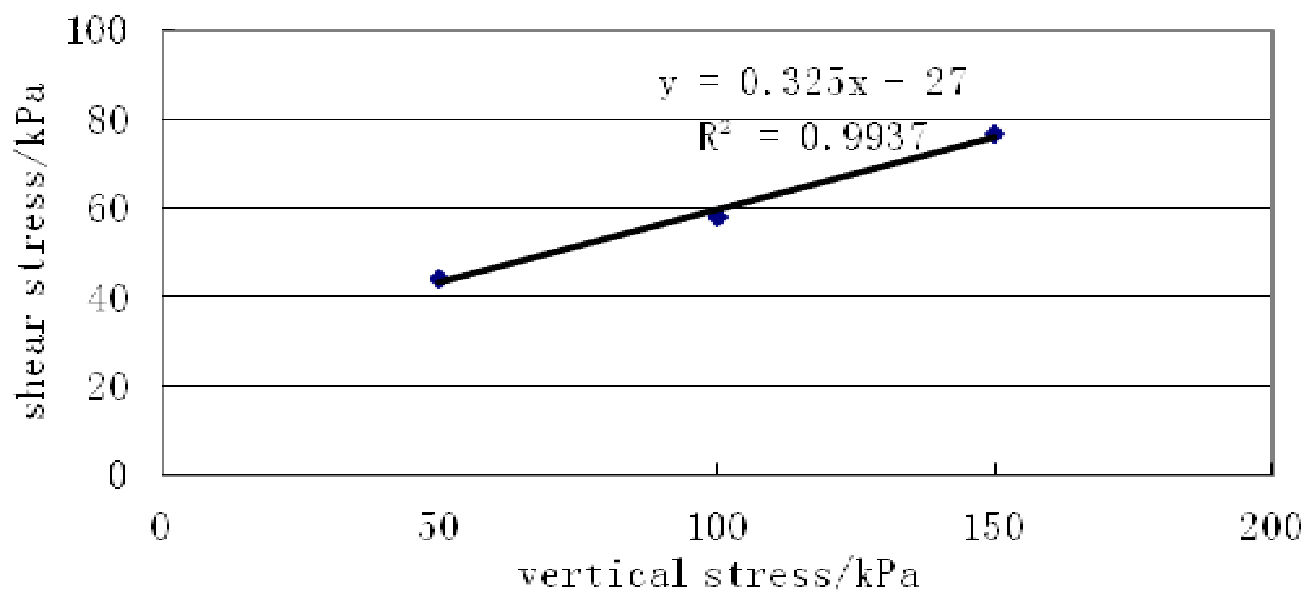

(b) The first time wetting-drying cycle 


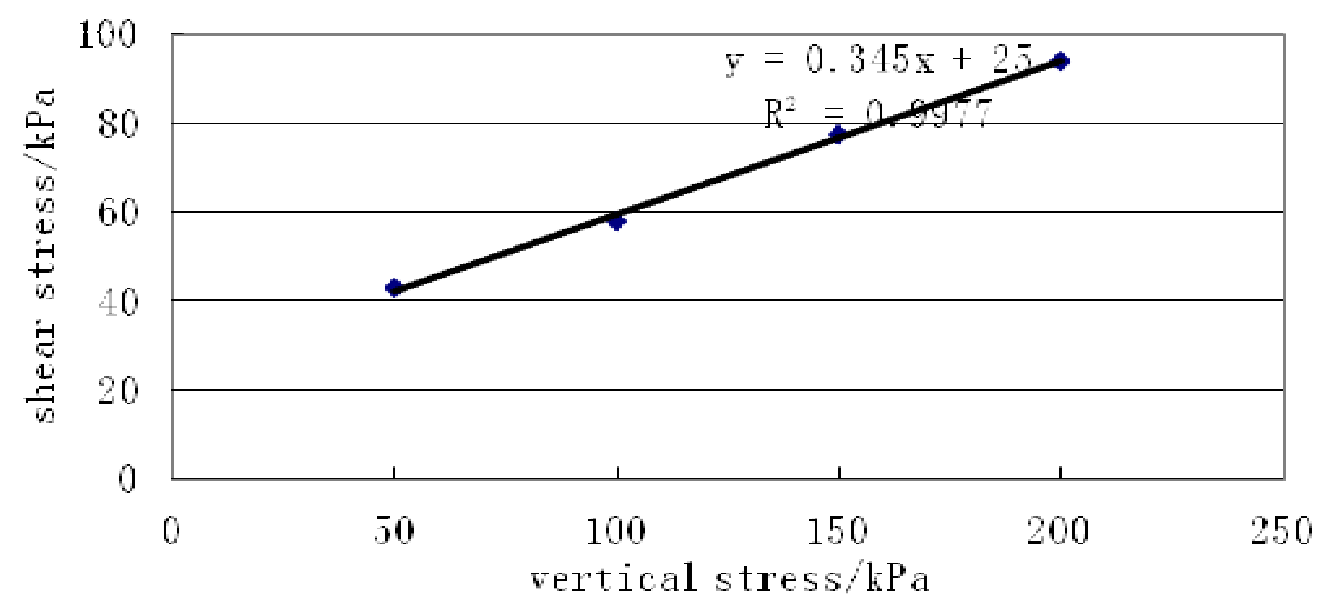

(c) The second time wetting-drying cycle

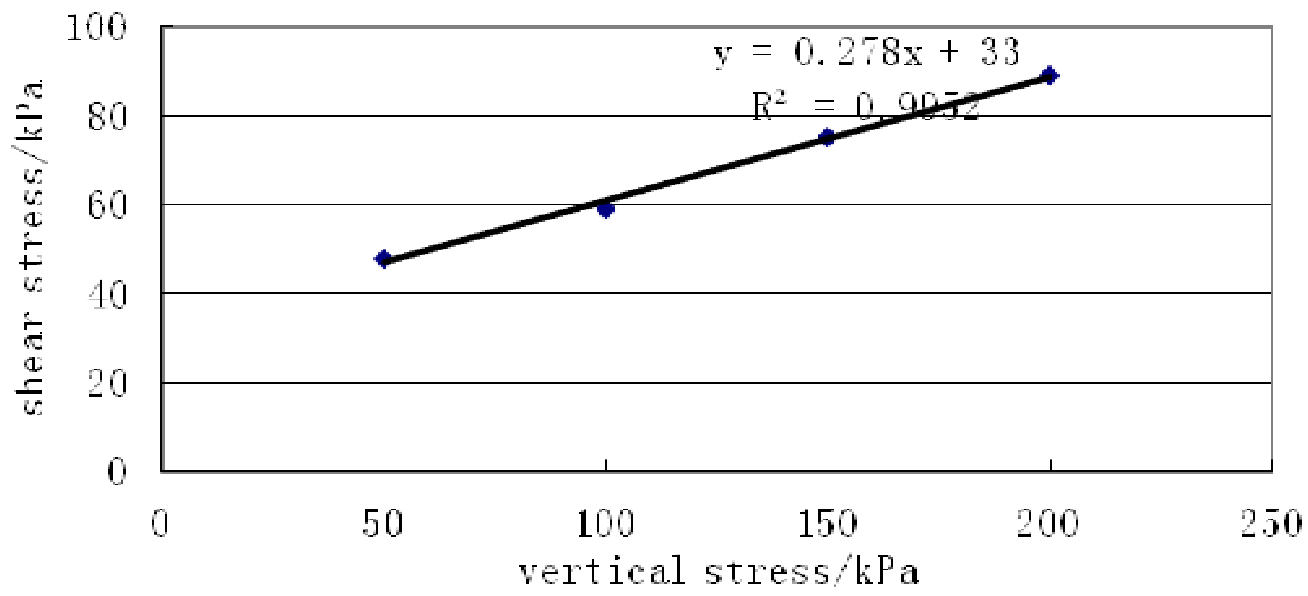

(d) the third time wetting-drying cycle

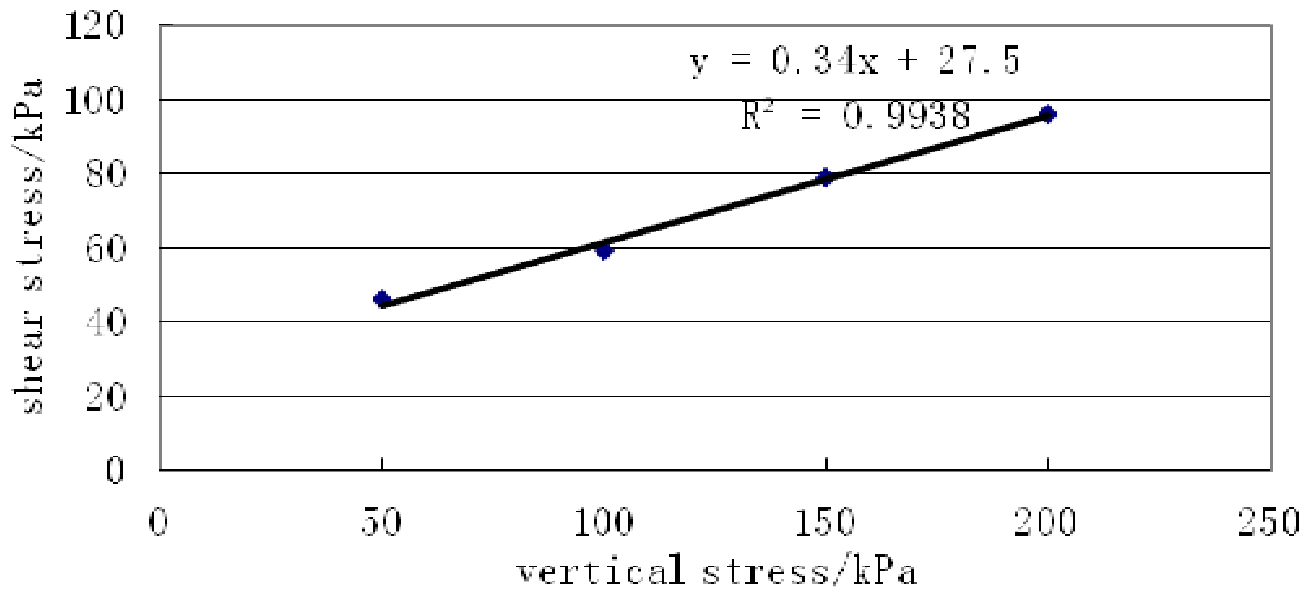

(e) the fourth time wetting-drying cycle

Fig.1 the relationship between vertical stress and shear stress of expansive rock under different wetting-drying cycle times 


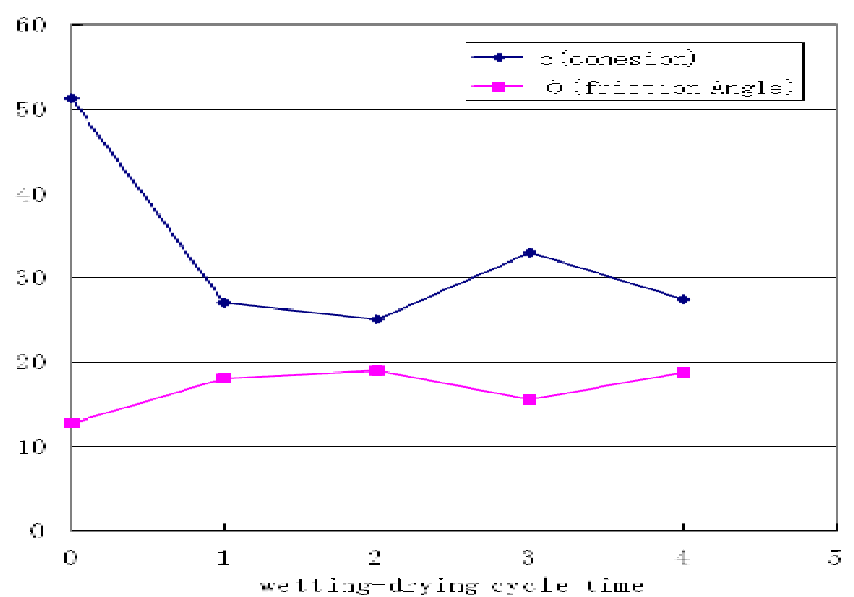

Fig. 2 the variation of the index of the strength of the expansive rock shear strength with the wetting-drying cycle times

From the Fig. 1 and Fig. 2, it could be seen that, the relationship between the cohesion, the friction Angle and the wetting-drying cycle times was difficult to described by using a certain function. But the overall trend was more obvious. After the first wetting-drying cycle, the cohesion $\mathrm{c}$ attenuated by $37.1 \%$. After the second wetting-drying cycle, the attenuation was small, but the value was slightly increased after the third cycle. But the overall strength was less than the samples which has no wetting-drying cycle. The friction Angle was the lowest in the zero cycle, followed by the wetting-drying cycles, the value of which was repeatedly raised and lowered, but the total value doesn't show a great variation.

\section{Conclusions}

The in-room wetting-drying test of expansive rock samples indicates that the cohesion c decreases greatly after the first wetting-drying process. When it refered to the design and the stability analysis for the slope, the influence of cyclic wetting-drying test on the strength characteristics of expansive rock must be considered.

\section{Acknowledgements}

This work was financially supported by the Hubei Natural Science Foundation (2015CFC831) and Key Laboratory of Geological Hazards on Three Gorges Reservoir Area(China Three Gorges University),Ministry of Education(2015KDZ17).

The Corresponding author is ZHAO Erping.

\section{References}

[1] Zeng Jijie.Research on the stability of expansive rock slope in the Nanning basin[D].Guangxi: Guangxi university,2004.12.

[2] Zang Deji,Liu Xinhong,Wang Bin.Study on Shear Behavior of Undisturbed Expansive Rock by Direct Shear Test[J]. Chinese Journal of Underground Space and Engineering,2009,5(5):915-919.

[3] He Xiaomin,Xu Yanyong,Huang Bin,Yang Hong,Xu Han. Swelling Rock in Middle-Route of Soutll-to-North Water Transfer Project [J.]South-to-North Water Transfers and Water Science \& Technology,2008,6(11):38-47.

[4] Hou Xuemei. Study of the Basic Characteristics of Expansive Rock and its Rapid protective Materials [D].Wuhan: Changjiang RIVER scientific research institute 2009.6.1.

[5] Xu Han, Huang Bin, He Xiaomin. Experimentation study on engineering property of swelling rocks[J].SHUI LI XUE BAO,2007,10:716-722. 